\title{
Known unknowns for allele-specific expression and genomic imprinting effects Christopher Gregg ${ }^{1,2}$
}

\author{
Addresses: ${ }^{1}$ Department of Neurobiology \& Anatomy and Human Genetics, University of Utah School of Medicine, 323 Wintrobe Bldg 530 \\ University of Utah, School of Medicine, 20 North 1900 East, Salt Lake City, UT 84132-3401 USA; ${ }^{2}$ The New York Stem Cell Foundation, \\ 178 Columbus Avenue \#237064, New York, NY 10023, USA \\ Email: chris.gregg@neuro.utah.edu \\ Fl000Prime Reports 2014, 6:75 (doi:10.12703/P6-75) \\ All F1000Prime Reports articles are distributed under the terms of the Creative Commons Attribution-Non Commercial License \\ (http://creativecommons.org/licenses/by-nc/3.0/legalcode), which permits non-commercial use, distribution, and reproduction in any medium, \\ provided the original work is properly cited. \\ The electronic version of this article is the complete one and can be found at: http://f1000.com/prime/reports/b/6/75
}

\begin{abstract}
Recent studies have provided evidence for non-canonical imprinting effects that are associated with allele-specific expression biases at the tissue level in mice. These imprinting effects have features that are distinct from canonical imprinting effects that involve allele silencing. Here, I discuss some of the evidence for non-canonical imprinting effects in the context of random $\mathrm{X}$-inactivation and epigenetic allele-specific expression effects on the autosomes. I propose several mechanisms that may underlie non-canonical imprinting effects and outline future directions and approaches to study these effects at the cellular level in vivo. The growing evidence for complex allele-specific expression effects that are cell- and developmental stage-specific has opened a new frontier for study. Currently, the function of these effects and the underlying regulatory mechanisms are largely unknown.
\end{abstract}

\section{Introduction}

Allele-specific expression (ASE) effects that arise due to epigenetic regulatory factors have previously been thought to be relatively rare in the genome. For example, the ASE effects associated with clusters of imprinted genes, olfactory receptors, protocadherins, and random $\mathrm{X}$-inactivation have been considered exceptions to the rules of Mendelian inheritance. However, as I review below, new genomics-based approaches suggest that complex and tissue-specific ASE effects are prevalent in the genomes of mice and humans. Currently, these phenomena are both functionally and mechanistically poorly understood. Some of these ASE effects have been classified as random, whereas others have been classified as imprinting effects. Here, I discuss recent findings that challenge the concept of randomness in some cases and that suggest that complex parent-of-origin effects can influence ASE in a manner that has features distinct from those of canonical genomic imprinting. These new studies suggest that there are complex ASE effects that differ according to cell type and developmental stage and that exhibit variance between different individuals. In some cases, these effects appear to be influenced by the parental origin of the allele. I highlight new findings that are changing our understanding of allele-specific gene expression effects, and present a potential opportunity for the development of new therapeutic strategies that activate healthy alleles or silence mutated alleles at specific loci [1].

\section{Defining allele-specific gene expression effects} I define ASE effects to include any phenomenon that results in differences in the relative expression of a maternal versus paternal allele (Figure 1). These effects potentially encompass (a) allele-specific silencing effects, in which one allele is completely silent; (b) ASE biases, in which a significantly higher level of expression arises from one allele versus the other; and (c) allele-specific isoforms, in which differences exist in the relative expression of specific transcripts from one allele versus the other. Each of these ASE effects can be further subcategorized depending upon whether the effects vary by cell type and developmental stage and between individuals. Furthermore, in each of these contexts, a parent-of-origin effect arises whenever a significant bias to 
Figure I. Subtypes of allele-specific expression effects

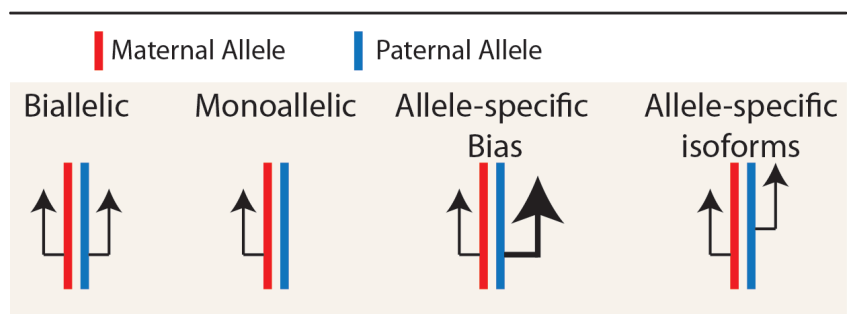

Allele-specific expression effects could potentially take on four basic forms that involve ( $\mathrm{I}$ ) equal expression of the maternal (red) and paternal (blue) alleles (biallelic), (2) complete silencing of one allele (monoallelic), (3) a bias to express either the maternal or paternal allele at a higher level (allele-specific bias), or (4) the expression of different isoforms from the maternal versus the paternal allele (allele-specific isoforms).

express the maternal or paternal allele exists across a population of individuals. These categories provide testable models to explore the concept of randomness in ASE effects, as I discuss below.

\section{Parent-of-origin effects and random $\mathbf{X}$-inactivation}

$\mathrm{X}$-inactivation in female somatic tissues is considered by many to be a classic example of a random monoallelic effect because either the maternally (Xm) or paternally (Xp) inherited $\mathrm{X}$ chromosome is randomly inactivated in each cell [2]. The decision to express the Xm or Xp is typically thought to be a stochastic process that occurs in precursor cells, giving rise to a variegated pattern of $\mathrm{Xm}$ - and Xp-expressing cells in mature female somatic tissues. However, if X-inactivation is random in all cases, a problem appears to arise for X-linked imprinted genes. $\mathrm{X}$-linked imprinting effects that act at the gene level and that result in the differential expression of specific genes on the Xm versus the Xp have been defined in mice. These effects have been shown to be both developmental stage- and tissue-specific. It is unclear how such effects could be functional and selected for if X-inactivation is randomly variegated in all tissues, like the coat of a calico cat. Below, I expand on these issues, describe studies that suggest that $\mathrm{X}$-inactivation is not random in mouse somatic tissues in some cases, and propose that parentof-origin effects that can bias X-inactivation provide a consistent background from which X-linked imprinted genes may function.

Currently, six X-linked imprinted genes have been discovered in mice. Genes that are preferentially expressed from the Xp include Xist and Rhox5 [3,4], and genes that are expressed from the Xm include $X l r 3 b, X l r 3 c$, $\mathrm{Xlr} 4 \mathrm{~b}$, and $\mathrm{Xlr} 4 \mathrm{c}[5,6]$. Interestingly, the imprinted $\mathrm{Xlr}$ genes are highly expressed in the brain and have been proposed as a potential mechanism for X-linked parentof-origin effects on behavior [5]. Imprinting for the Xlr genes is specific to brain region, tissue, and the developmental stage [6]. For example, Xlr3b exhibits biallelic expression (equal expression from the Xm and $\mathrm{Xp}$ ) in the midbrain and hindbrain at E14.5 but is paternally repressed in these regions after birth. In the liver, the paternal $X l r 3 b$ allele is strongly repressed, but the paternal $X l r 4 b$ allele exhibits weak repression (a maternal bias). In the brain, the paternal allele for both genes is strongly repressed and expression arises exclusively from the Xm. Thus, X-linked imprinting effects at the gene level can be highly tissue - and developmental stage -specific. How can these effects be functionally meaningful and selected for if the pattern of X-inactivation is patchy and random between individuals?

One potential outcome from random X-inactivation could be that female tissues are composed of an equal number of Xm- and Xp-expressing cells that are relatively evenly distributed (Figure 2A). In this case, each individual would have a substantial $\mathrm{Xm}$ and $\mathrm{Xp}$ contribution to a given structure, and thus the effects of $X l r$ imprinting would be similar between different individuals. However, an elegant new study by Wu and colleagues [7] demonstrates that this is not the case. The authors used an X-linked reporter system in combination with cell type-specific Cre drivers to systematically analyze X-chromosome inactivation patterns in different tissues and cell types. They conclude that X-inactivation is highly variable between individuals. Even within litters, extreme variation was detected such that some littermates express primarily the $\mathrm{Xp}$, whereas others express primarily the Xm. In their analysis, they found entire regions of the brain or other tissues that were populated primarily by cells that chose one $\mathrm{X}$ over the other. This extremely variegated pattern seems at odds with functional roles for $\mathrm{X}$-linked imprinted genes, as described above. For example, the $\mathrm{Xlr}$ genes are preferentially expressed from the $\mathrm{Xm}$; thus, if different regions of the brain are composed primarily of $\mathrm{Xm}^{+}$cells in one individual but of $\mathrm{Xp}^{+}$cells in another individual (Figure $2 \mathrm{~B}-$ as found in the study by $\mathrm{Wu}$ and colleagues), then the effect of the gene expression program that is unique to Xm-expressing cells would be extremely variable between individuals, seemingly precluding selective effects and functional roles for the imprinting. A potential solution to this could arise if X-inactivation was biased in favor of the expression of the $\mathrm{Xm}$ for tissues in which the $\mathrm{Xlr}$ imprinting effects function (for example, in the brain). The bias would effectively buffer against extreme variation between individuals and provide a more consistent background for Xlr imprinting effects (Figure 2C). The study by Wu and colleagues did not find any evidence for 
Figure 2. The relationship between $X$-linked imprinted genes and random $X$-inactivation patterns

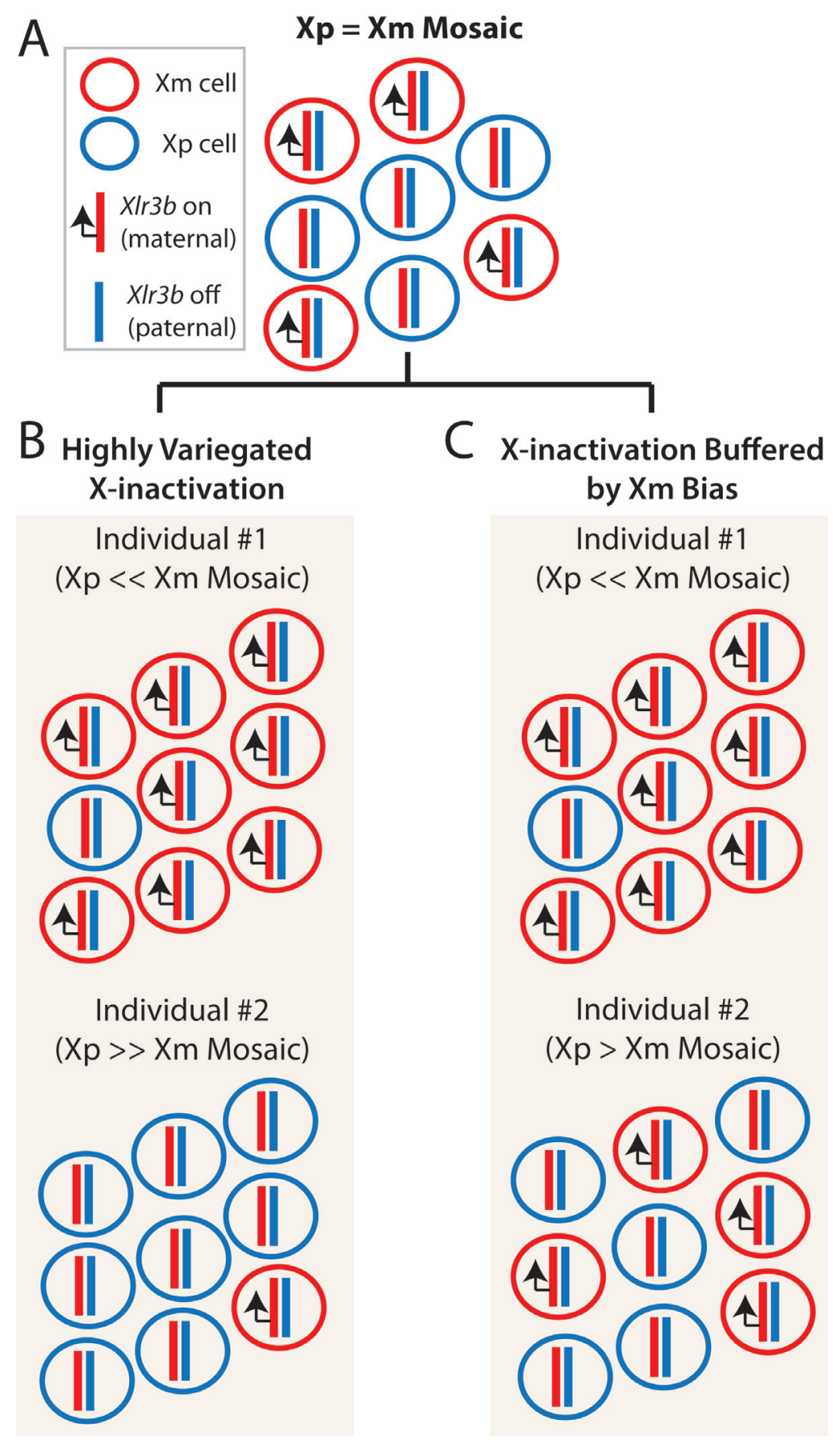

This schematic illustrates the relationship between different $X$-inactivation patterns and $X$-linked imprinted genes, such as $X \mid r 3 b$, which is an $X$-linked imprinted gene that is preferentially expressed from the $X m$ allele. (A) If random $X$-inactivation in female tissues resulted in an equal number of $X m$ and $X p$ expressing cells, then X-linked imprinted genes like XIr3b would have a consistent effect on different individuals. However, this pattern does not occur. (B) The study by Wu and colleagues [7] uncovered highly variable $X$-inactivation patterns in different tissues and individuals. This random and variegated pattern results in some brain regions or tissues being primarily populated by $\mathrm{Xm}^{+}$cells in one individual and $\mathrm{Xp}^{+}$cells in another. This pattern would introduce extreme variance for the effects of X-linked imprinted genes, like XIr3b, in a population of individuals. (C) A parent-of-origin effect that biases $X$-inactivation toward the Xm could potentially buffer the variance associated with random $\mathrm{X}$-inactivation, such that a minimum contribution of $\mathrm{Xm}^{+}$cells exists in tissues where $X I r 3 b$ imprinting functions, such as the brain. In this scenario, pronounced $X m$ biases would still arise, perhaps increasing the impact of $X \mid r 3 b$ imprinting effects (individual \#I), but the $X m$ inactivation bias would buffer against extreme $X p$ biases that could negate the effects of $X I r 3 b$ imprinting (individual \#2). 
parent-of-origin effects that bias X-inactivation toward the expression of the Xm in the brain, nor did they find any bias in any of the other cell types or tissues they analyzed. However, others have reported these effects, as described below.

Genomic imprinting effects that influence X-inactivation are known to occur in mice in the zygote, when the Xp is specifically silenced because of a paternally inherited imprint. This imprinting effect is maintained in extraembryonic tissues. In contrast, at the epiblast stage in the embryo proper, the $\mathrm{Xp}$ is reactivated and random $\mathrm{X}$ inactivation occurs [8]. However, using an RNA-Seq-based approach in F1 hybrid mice, we and others detected an overall bias to express the Xm over the $\mathrm{Xp}$ in the adult female mouse brain $[9,10]$. These independent studies used different mouse strains, suggesting that the effect is reproducible in multiple genetic backgrounds. Our study found further support for the Xm bias in the brain by using an X-linked enhanced green fluorescent protein (EGFP) transgenic reporter line [9]. These results support earlier work that first uncovered parent-of-origin effects influencing X-inactivation in the embryo [11-14], and the results were recently replicated [15].

Interestingly, parent-of-origin effects that bias X-inactivation patterns can be highly cell type-specific. A recent study of mouse mammary tissues revealed that the $\mathrm{Xp}$ is preferentially expressed over the $\mathrm{Xm}$ in mouse alveolar cells [16]. The authors found that a paternally inherited deletion of the X-linked gene Rnf12, a really interesting new gene (RING) finger LIM domain-interacting protein, inhibits alveolar differentiation and milk production in pregnant female offspring. No effect was observed when the mutated allele was maternally inherited. Using the same $\mathrm{X}$-linked EGFP reporter line indicated above as well as an analysis of X-linked ASE patterns in hybrid mice, the authors were able to demonstrate that the $\mathrm{Xp}$ is preferentially expressed in alveolar cells, thus explaining the parent-oforigin effect. In summary, parent-of-origin effects that bias random X-inactivation in mice have been identified by multiple studies, but it is not yet clear how to reconcile these observations with the highly variegated and random $\mathrm{X}$-inactivation patterns described by $\mathrm{Wu}$ and colleagues. I propose that an important study of different alleles of the X-inactivation control element (Xce) in mice provides some possible insights [15].

This recent study of the Xce defined the relative strength and identity of six different Xce alleles $\left(X c e^{a}, X c e^{b}, X c e^{c}\right.$, $X c e^{d}, X c e^{e}$, and $\left.X c e^{f}\right)$ through genetic crosses conducted with 10 different mouse strains. The authors found an $\mathrm{Xm}$ expression bias in most crosses and propose that the parent-of-origin effects that influence X-inactivation are encoded in an undefined region outside of the Xce. However, the authors also discovered that parent-of-origin effects on X-inactivation do not occur in crosses involving the $X c e^{a}$ and $X c e^{b}$ alleles. The weak $X c e^{a}$ allele is present in the $129 / \mathrm{Sv}$ mouse strain, and the $X c e^{b}$ allele is present in C57BL/6J mice. Importantly, 129/Sv and C57BL/6J hybrids are commonly used for gene targeting and the generation of transgenic mice. Indeed, the background for the targeted transgenic reporter lines generated by $\mathrm{Wu}$ and colleagues to map X-inactivation patterns is reported as a $129 / \mathrm{Sv}$ x C57BL/6J mixed background. Thus, the lack of parent-of-origin effects on X-inactivation in these lines may be related to the genetic background of the mice.

Further studies focusing on the relationship between the genetic background and parent-of-origin effects influencing X-inactivation are warranted. It is of interest to determine whether Mus subspecies that lack parent-oforigin influences on X-inactivation also lack imprinting effects for genes, such as the Xlr genes. If a relationship exists between these two phenomena, then the problem of how X-linked imprinted genes evolved in the face of seemingly stochastic and highly variegated inactivation patterns might be resolved by the existence of a parental bias that buffers random X-inactivation and provides a stable background for X-linked imprinted genes, such as the $\mathrm{Xlr}$ genes (Figure 2C). A major point that emerges from the studies presented above is that parent-of-origin effects influence random X-inactivation in mice-the prototypical example of a random allelic effect-and I propose a potential functional role for this effect. Next, I discuss the potential for parent-of-origin effects to influence random monoallelic expression effects on the autosomes, which are now known to be widespread in mice and humans.

\section{Autosomal random monoallelic expression effects}

Widespread random monoallelic expression (RMAE) effects influence approximately $20 \%$ of genes in the genome and were first described by Gimelbrant and colleagues [17] in clonally derived human lymphoblastoid cell lines. Subsequently, numerous studies have provided supporting evidence for the prevalence of RMAE effects in cell lines derived from mice and humans [18-23]. Furthermore, Nag and colleagues [24] defined an epigenetic signature for genes that exhibit random monoallelic effects, which consists of the simultaneous appearance of chromatin marks for active transcription (H3K36me3) and transcriptional silencing (H3K27me3) in a gene body. This signature is present for $20 \%$ of ubiquitously expressed genes and $30 \%$ of tissue-specific genes in humans. RMAE effects are stable over multiple cell divisions and distinct from other forms of ASE effects. These pioneering studies have fundamentally changed our understanding of gene 
expression. However, little is known about the nature of these effects in vivo or the potential for parent-of-origin effects to influence random ASE.

Typically, studies of RMAE effects involve deriving five to eight clonal cell lines that are propagated in vitro. Genes exhibiting RMAE effects have been defined as those that exhibit variance between different clones in terms of the number of alleles and identity of the allele that is expressed for a given gene [25]. The apparently stochastic nature of this outcome in clones from the same cell type propagated under similar culture conditions presents a challenge in terms of defining the functional significance of the effects and the underlying mechanisms. Two recent studies of RMAE effects in embryonic stem cells (ESCs) that were differentiated into neural precursor cells (NPCs) revealed that the number of genes exhibiting RMAE effects increases by over fivefold as a consequence of differentiation $[20,22]$. Thus, changes to ASE effects are associated with cellular lineage commitment, and RMAE effects appear to be highly tissue- and cell typespecific. Interestingly, in addition to the analysis of cell lines, the study by Gendrel and colleagues [22] analyzed allele expression effects in vivo by using in situ probes targeting nascent RNAs. The authors report that all RMAE candidate genes analyzed exhibit monoallelic expression in at least a subpopulation of cells in vivo. Of note, many biallelic control genes also exhibited monoallelic expression in a substantial subpopulation of cells in vivo (approximately $20 \%$ to $40 \%$ of cells). The relative proportions of monoallelic and biallelic cells varied in different tissues for different genes. For example, Eya1 exhibits monoallelic expression in essentially all expressing cells in the adult lens but in only $50 \%$ of expressing cells in the developing kidney. Overall, these early studies demonstrate that ASE effects are common in vitro and in vivo.

Given the small number of clones examined and the approaches used in these studies, it has been difficult to determine whether any RMAE genes exhibit a significant bias favoring the paternal versus the maternal allele when an allele-specific silencing event occurs. In vivo, the parental bias could arise in a highly specific cell type, similar to the Xp bias that occurs in mouse alveolar cells (see above). In a recent genome-wide study of RMAE effects in pre-B lymphoblastoid cell lines from hybrid mice, the authors provided evidence for allele "skewing" effects, in which the maternal and paternal alleles are unequal in terms of their probability of being silenced when a monoallelic expression event occurs [18]. The study examined RMAE genes that had more than one clone in which an allele-specific silencing event occurred and counted the number of times the same allele was selected. The study did not examine sufficient clones to statistically define skewing effects at the gene level; however, the authors demonstrated that skewing effects in their data are consistent with one of two models. Either $40 \%$ of random monoallelic genes exhibit a strict preference for one allele, or $80 \%$ of random monoallelic genes prefer one parental allele $85 \%$ of the time. Similarly, in their study of RMAE effects in NPCs, Gendrel and colleagues [22] noted that for many genes exhibiting monoallelic effects, one allele was preferentially expressed. Neither study was able to determine whether the skewing effects involve a parent-of-origin effect due to the absence of a reciprocal cross and the limited number of clones examined.

In summary, we know little about whether parent-oforigin effects can influence RMAE effects on the autosomes. Rather than advocating for more in vitro studies, I believe that the potential for parent-of-origin influences to bias ASE effects is better studied in vivo (at least in mice). Parent-of-origin effects that bias or silence the expression of one parental allele over the other in a subpopulation of cells only could emerge as allelespecific expression biases at the tissue level. Interestingly, some studies of genomic imprinting effects have detected these biases, as I detail below.

\section{Non-canonical autosomal imprinting effects}

Canonical genomic imprinting involves allele-specific silencing effects that do not vary between healthy individuals in mice $[26,27]$. Most imprinted genes reside in clusters, of which there are a total of 16 in the mouse. Canonical imprinting is mechanistically defined by the effects of differentially methylated regions (DMRs) in the genome that are located at specific sites, called imprinting control regions (ICRs) $[26,27]$. ICRs are cis-acting elements that regulate imprinting at nearby genes, resulting in the formation of imprinted gene clusters in most cases. In total, 55 DMRs have been identified in the mouse genome [28]. Maternally methylated DMRs are located at promoters and can regulate the expression of long non-coding RNAs that regulate imprinting effects for neighboring genes. Paternally methylated DMRs are located in intergenic regions and have been shown to regulate distinct promoter-enhancer interactions on maternal and paternal chromosomes. Several proteins that play essential roles in the establishment of DMRs in the parental gametes and the maintenance of these marks in the embryo have been defined and recently reviewed [26,27]. Importantly, all of the genes that exhibit the canonical imprinting effects I have detailed here have been identified in the mouse genome. However, although canonical imprinted genes exhibit allele silencing, a growing list of genes in the genome 
have been shown to exhibit biases to express either the maternal or paternal allele and much less is known about the frequency and nature of these effects.

An essay by Khatib first drew attention to the fact that some imprinted genes exhibit allele-specific expression biases rather than allele-silencing [29]. However, the phenomenon has largely been overlooked. Previously, we and others performed genome-wide screens for imprinting effects in brain $[9,30-32]$ or placental tissues [33] by using RNA-Seq analyses of the transcriptome of reciprocal F1 hybrid mice. Single-nucleotide variants in the RNA-Seq data were used to distinguish expression from the maternally versus paternally inherited allele for each expressed gene. The novel "imprinted genes" uncovered in these studies typically involved significant maternal or paternal allele-specific expression biases rather than the canonical genomic imprinting effects defined above. For example, Casd1, Pde4d, Bcl2l1, Cobl, $D d c, M r p l 48$, and $I l 18$ were found to exhibit imprinting effects in the brain, and in all cases an allele-specific expression bias rather than allele silencing was found $[9,30,31]$. Here, I refer to these effects as non-canonical imprinting effects since the phenomenon involves allele expression biases rather than the allele silencing that is typically associated with canonical imprinting. In the case of $D d c$, canonical imprinting occurs in the heart involving the expression of the paternal allele [34], whereas a non-canonical imprinting effect was found in the brain involving a bias to express the maternal allele [30]. The number of genes that exhibit non-canonical imprinting effects in the genome is currently debated. Some RNA-Seq-based surveys of imprinting have found very little evidence for novel imprinting effects [31,32], whereas others have suggested that the effects are more widespread [30,33]. The studies by Gregg and colleagues $[9,30]$ in which many novel imprinting effects were uncovered have been shown to have a high false discovery rate, and the conclusion that many noncanonical imprinting effects exist in the genome has been challenged [35]. However, it is unlikely that published screens for non-canonical imprinting effects have reached saturation, particularly for effects that are similar in magnitude to the parent-of-origin effects that influence X-inactivation (Huang et al., unpublished data). The detection of the Xm bias in the female brain by RNA-Seq was achieved by pooling data across all X-linked genes $[9,10]$. These studies were not sufficiently powered to statistically detect the maternal bias for individual X-linked genes. Indeed, little attention has been paid to type II statistical error rates in published surveys of imprinting effects. Emerging studies that more effectively manage type I and type II statistical errors will provide important insights into the nature and prevalence of non-canonical imprinting effects. A new study of genomic imprinting effects in the mouse placenta, for example, suggests that non-canonical imprinting effects may influence the expression of many genes in this tissue [36].

In the above study by Finn and colleagues [36], the authors performed RNA-Seq to analyze ASE in F1 hybrid mice derived from reciprocal matings of Cast x C57BL/6J mice. Their study used large numbers of biological replicates ( $\mathrm{n}=11$ to 12 for each cross) and an RNA-Seqbased approach called 3SEQ, which specifically targets the $3^{\prime}$ end of transcripts. The study revealed 78 novel imprinted genes in the placenta, some of which were independently validated by using pyrosequencing. All of these genes exhibit an allele-specific expression bias rather than canonical imprinting. A maternal bias was detected for 75 out of the 78 novel genes and beyond these high confidence candidates, the authors provide further evidence that hundreds of transcripts exhibit maternal expression biases in the E11.5 placenta. A concern is that these biases arise due to maternal decidual contamination; however, the authors performed multiple controls that suggest that this contamination is unlikely to explain their results. The widespread maternal effect was developmental stage- and tissue-specific, as it was not present in the yolk sac or the E17.5 placenta. Finally, the authors analyzed individual offspring from multiple litters in their study, which allowed them to discover that the maternal non-canonical imprinting effects in their data can vary according to litter. These results suggest that non-canonical imprinting effects are more widespread and dynamic in the placenta than was previously appreciated. These effects contrast sharply with the relatively stable and robust allelesilencing effects associated with canonical imprinted genes in somatic tissues.

The nature of non-canonical imprinting effects at the cellular level in vivo is unknown, and multiple models might explain the effects. One testable hypothesis is that some non-canonical imprinting effects reflect allelespecific silencing events in a subpopulation of cells. Under this model, a tissue is composed of a mosaic of cells in which some cells express both alleles for a given non-canonical imprinted gene and some express one specific parental allele (Figure 3A), or all cells exhibit monoallelic expression, but the decision to select the maternal or paternal allele is skewed such that a parental bias emerges at the level of a cell population (Figure 3B). The rationale for these models emerges from both the RMAE field and the imprinting field. Cell type-specific imprinting effects are known to occur. For example, Dlk1 is imprinted in most cells that express the gene in the 
Figure 3. Potential parent-of-origin effects on allele-specific expression at the cellular level

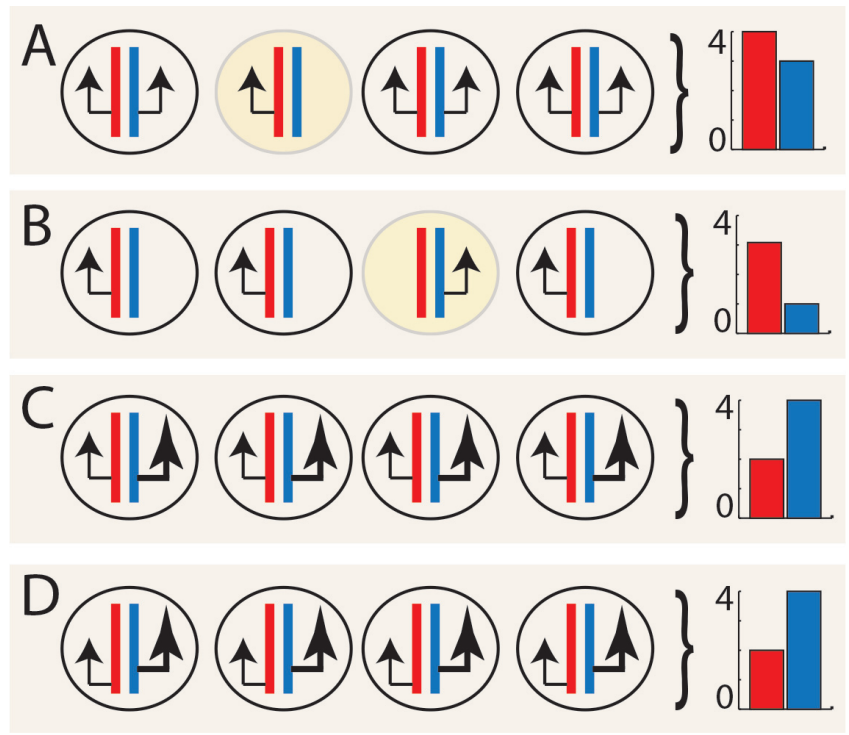

This schematic illustrates different models by which non-canonical imprinting effects, which involve biases to express either the maternal or paternal allele at the tissue level (bar plots; red, maternal allele expression; blue, paternal allele expression), could emerge at the cellular level. (A) An allele-specific expression bias could emerge because of silencing of one parental allele in a subpopulation of cells. (B) A bias could emerge because of random monoallelic expression effects that are skewed toward the selection of one parental allele over the other. (C) A bias to express one parental allele over the other could exist in all or most cells expressing the non-canonically imprinted gene. (D) The non-canonical imprinting effect could arise at the gene level because of the expression of distinct and overlapping isoforms from the maternal versus the paternal allele, with one isoform expressed at a higher level.

brain with the exception of neural stem cells of the subventricular zone, which express both alleles [37]. Additionally, Ube $3 a$ imprinting occurs in differentiated neurons, but not glia $[38,39]$, and the canonical imprinted genes Grb10, Igf2, and Igf2r also exhibit distinct imprinting patterns in different cell types [40-43]. Thus, even among canonical imprinted genes, cell- and tissue-specific imprinting effects exist. In addition, a preliminary analysis of recent studies of in vitro RMAE effects indicates a relationship to imprinting in a few cases. For example, Mrpl48 exhibits a random allele-specific expression bias in NPCs analyzed in vitro by Gendrel and colleagues [22] but exhibits a non-canonical imprinting effect involving a paternal bias in the female hypothalamus $[9,30]$. In addition, we previously identified and validated noncanonical imprinting effects for genes within the protocadherin gamma cluster [30], which have also been shown to exhibit RMAE effects [44]. Finally, some genes found to exhibit RMAE effects in different NPC clones by the studies by Gendrel and colleagues [22] or Eckersley-Maslin and colleagues [20] (or both) are known imprinted genes, such as Cdkn1c, Airn, Sgce, Mcts2, and H13. Thus, in some cases, genes that exhibit RMAE effects in vitro exhibit imprinting effects in vivo.

Non-canonical imprinting effects detected at the tissue level could also arise due to allele-specific expression biases at the cellular level, such that each cell expresses slightly more of one parent's allele (Figure 3C). The study of RMAE by Gendrel and colleagues [22] identified clones in which there was an allele-specific expression bias rather than strict monoallelic or biallelic expression, indicating that such effects can occur at the cellular level. These effects could be functionally significant if allelespecific biases occur for multiple genes in the same pathway or network, such that the parental biases become additive at the system level. A final hypothesis to consider is that some allele biases emerge at the gene level because of isoform-specific imprinting effects in which distinct but overlapping transcripts are differentially expressed from the maternal versus paternal allele (Figure 3D). Indeed, a few examples of isoform-specific imprinting events have been defined by using conventional polymerase chain reaction or RNA-Seq-based approaches for genes, such as H13, Herc3, Blcap, and Inpp5f [30,45-47]. Once the field has a detailed understanding of the genes that exhibit non-canonical imprinting effects in different somatic and extra-embryonic tissues, we will be able to test these hypotheses and begin to define specific subtypes of non-canonical imprinting based on the underlying mechanisms.

The fact that ASE effects change during cellular differentiation $[20,22]$ and that some canonical and non-canonical imprinting effects appear to change during differentiation [37] or development [30,36] suggests the possibility that allele-specific regulatory mechanisms may function to coordinate specific gene expression programs and influence cell fate decisions. A role for differential allelic regulation in cell fate decisions was previously observed for Nanog [48]. Furthermore, studies of RMAE effects have recently provided evidence that monoallelic expression effects are associated with reduced expression and could function to influence gene expression levels [22]; only a few genes that compensate for the loss of one allele have been identified [20]. Finally, one mechanistic model for non-canonical imprinting involves DMRs that are functional in a specific subpopulation of cells. However, a recent survey of DMRs in the brain found few novel DMRs outside of known imprinted gene clusters [28]. Thus, this mechanism is unlikely unless a cell type-specific analysis of DMRs is required to detect them. Interestingly, other mechanisms that influence ASE effects may exist. Studies 
of RMAE effects suggest that allele silencing might not require DNA methylation and that some other mechanism for stable allele-specific silencing remains to be defined $[20,22,48]$.

\section{Outlook}

Genetic studies in mice clearly indicate that parent-oforigin effects influence several complex traits $[49,50]$. However, many mechanisms and details remain to be understood, and the function of imprinting is debated. Here, I have discussed some observations in the ASE field that provide evidence for non-canonical imprinting effects involving maternal or paternal biases that influence random X-inactivation and the allelic expression of some autosomal genes. It is likely that published RNA-Seq-based screens for imprinting are not saturated for the detection of non-canonical imprinting effects, and in the light of the discovery that ASE effects are widespread and arise in subpopulations of cells in vivo, a deeper investigation into the nature and function of non-canonical imprinting effects in different tissues and cell types in vivo is warranted. Several models might explain the detection of these effects at the tissue level, including maternally or paternally biased RMAE effects, overlapping transcripts, and cell type-specific imprinting. The recent developments in the ASE field have exposed many questions and opened an exciting frontier that is likely to advance our understanding of the genetic and epigenetic factors that influence phenotypes and disease susceptibility. An important question that I have not touched on is the possibility that ASE effects can be influenced by physiological and environmental factors acting on parents or offspring or both. A challenge for the field involves the development of in vivo approaches to further analyze allele-specific expression effects accurately in specific cell types in vivo, such as allele-specific in situ hybridization approaches that can discern the parental allele being expressed $[51,52]$ or RNA-Seq-based methods that permit the study of ASE effects in specific cell populations [19,5355]. Most enticing is the fact that the functions and regulatory mechanisms for many of these effects are currently unknown.

\section{Abbreviations}

ASE, allele-specific expression; DMRs, differentially methylated regions; EGFP, enhanced green fluorescent protein; ICR, imprinting control region; NPC, neural precursor cell; RMAE, random monoallelic expression; Xce, X-inactivation control element.

\section{Disclosures}

The author declares that he has no disclosures.

\section{Acknowledgments}

The author wishes to thank members of the Gregg lab and Alejandro Sánchez Alvarado, Jan Christian, and Thomas $\mathrm{O}^{\prime}$ Hare and the anonymous reviewers for the critical comments on an earlier version of this manuscript. The author is funded by the New York Stem Cell Foundation, the Simons Foundation Autism Research Initiative, and a University of Utah Seed Grant.

\section{References}

I. Huang H, Allen JA, Mabb AM, King IF, Miriyala J, Taylor-Blake B, Sciaky N, Dutton JW, Lee H, Chen X, Jin J, Bridges AS, Zylka MJ, Roth BL, Philpot BD: Topoisomerase inhibitors unsilence the dormant allele of Ube3a in neurons. Nature 2012, 48 I:I85-9.

\section{FlOOOPrime}

2. Lee JT: Gracefully ageing at $\mathbf{5 0 ,} \mathbf{X}$-chromosome inactivation becomes a paradigm for RNA and chromatin control. Nat Rev Mol Cell Biol 20II, I2:8I5-26.

3. Kobayashi S, Isotani A, Mise N, Yamamoto M, Fujihara Y, Kaseda K, Nakanishi T, lkawa M, Hamada H, Abe K, Okabe M: Comparison of gene expression in male and female mouse blastocysts revealed imprinting of the $X$-linked gene, Rhox $5 / P e m$, at preimplantation stages. Curr Biol 2006, 16:166-72.

4. Maclean JA, Bettegowda A, Kim BJ, Lou C, Yang S, Bhardwaj A, Shanker S, Hu Z, Fan Y, Eckardt S, McLaughlin KJ, Skoultchi Al, Wilkinson MF: The rhox homeobox gene cluster is imprinted and selectively targeted for regulation by histone $\mathrm{hl}$ and DNA methylation. Mol Cell Biol 20I I, 3 I:I275-87.

5. Davies W, Isles A, Smith R, Karunadasa D, Burrmann D, Humby T, Ojarikre O, Biggin C, Skuse D, Burgoyne P, Wilkinson L: XIr3b is a new imprinted candidate for $X$-linked parent-of-origin effects on cognitive function in mice. Nat Genet 2005, 37:625-9.

\section{FIOOOPrime}

RECOMMENDED

6. Raefski AS, O'Neill MJ: Identification of a cluster of $\mathbf{X}$-linked imprinted genes in mice. Nat Genet 2005, 37:620-4.

\section{FlOOOPrime}

RECOMMENDED

7. Wu H, Luo J, Yu H, Rattner A, Mo A, Wang Y, Smallwood PM, Erlanger B, Wheelan SJ, Nathans J: Cellular resolution maps of $\mathbf{X}$ chromosome inactivation: implications for neural development, function, and disease. Neuron 2014, 81:103-19.

\section{FlOOOPrime}

\section{RECOMMENDED}

8. Deng X, Berletch JB, Di Nguyen K, Disteche CM: X chromosome regulation: diverse patterns in development, tissues and disease. Nat Rev Genet 2014, 15:367-78.

9. Gregg C, Zhang J, Butler JE, Haig D, Dulac C: Sex-specific parentof-origin allelic expression in the mouse brain. Science 2010 , 329:682-5.

\section{FlOOOPrime}

\section{RECOMMENDED}

10. Wang $X$, Soloway PD, Clark AG: Paternally biased $\mathbf{X}$ inactivation in mouse neonatal brain. Genome Biol 2010, II:R79.

\section{FlOOOPrime} RECOMMENDED

II. Forrester LM, Ansell JD: Parental influences on $\mathbf{X}$ chromosome expression. Genet Res 1985, 45:95-100.

12. Fowlis DJ, Ansell JD, Micklem HS: Further evidence for the importance of parental source of the $X$ ce allele in $X$ chromosome inactivation. Genet Res 199I, 58:63-5. 
13. Chadwick LH, Willard HF: Genetic and parent-of-origin influences on $\mathbf{X}$ chromosome choice in $\mathbf{X c e}$ heterozygous mice. Mamm Genome 2005, 16:691-9.

14. Bittner RE, Popoff I, Shorny S, Höger H, Wachtler F: Dystrophin expression in heterozygous $\mathrm{mdx} /++$ mice indicates imprinting of $\mathbf{X}$ chromosome inactivation by parent-of-origin-, tissue-, strain- and position-dependent factors. Anat Embryol 1997, 195:175-82.

15. Calaway JD, Lenarcic AB, Didion JP, Wang JR, Searle JB, McMillan L, Valdar W, Pardo-Manuel de Villena, Fernando: Genetic architecture of skewed $\mathbf{X}$ inactivation in the laboratory mouse. PLoS Genet 2013, 9:el003853.

\section{FlOOOPrime}

16. Jiao B, Ma H, Shokhirev MN, Drung A, Yang Q, Shin J, Lu S, Byron M, Kalantry S, Mercurio AM, Lawrence JB, Hoffmann A, Bach I: Paternal RLIM/Rnf $\mathbf{2}$ is a survival factor for milk-producing alveolar cells. Cell 20I2, I49:630-4I.

\section{FlOOOPrime \\ RECOMMENDED}

17. Gimelbrant A, Hutchinson JN, Thompson BR, Chess A: Widespread monoallelic expression on human autosomes. Science 2007, 318: I136-40.

\section{FlOOOPrime \\ RECOMMENDED}

18. Zwemer LM, Zak A, Thompson BR, Kirby A, Daly MJ, Chess A, Gimelbrant AA: Autosomal monoallelic expression in the mouse. Genome Biol 20I2, I3:RI0.

\section{FlOOOPrime RECOMMENDED}

19. Tang F, Barbacioru C, Nordman E, Bao S, Lee C, Wang X, Tuch BB, Heard E, Lao K, Surani MA: Deterministic and stochastic allele specific gene expression in single mouse blastomeres. PLoS ONE 2011, 6:e21208.

20. Eckersley-Maslin MA, Thybert D, Bergmann JH, Marioni JC, Flicek P, Spector DL: Random monoallelic gene expression increases upon embryonic stem cell differentiation. Dev Cell 20I4, 28:35I-65.

\section{FlOOOPrime
RECOMMENDED}

21. Deng Q, Ramsköld D, Reinius B, Sandberg R: Single-cell RNA-seq reveals dynamic, random monoallelic gene expression in mammalian cells. Science 2014, 343:193-6.

\section{FIOOOPrime}

22. Gendrel A, Attia M, Chen C, Diabangouaya P, Servant N, Barillot E, Heard E: Developmental dynamics and disease potential of random monoallelic gene expression. Dev Cell 2014, 28:366-80.

\section{FIOOOPrime}

23. Li SM, Valo Z, Wang J, Gao H, Bowers CW, Singer-Sam J: Transcriptome-wide survey of mouse CNS-derived cells reveals monoallelic expression within novel gene families. PLOS ONE 2012, 7:e31751.

24. Nag A, Savova V, Fung H, Miron A, Yuan G, Zhang K, Gimelbrant AA: Chromatin signature of widespread monoallelic expression. Elife 2013, 2:e01256.

\section{FlOOOPrime}

\section{RECOMMENDED}

25. Chess A: Random and non-random monoallelic expression. Neuropsychopharmacology 2013, 38:55-6I.

26. Barlow DP, Bartolomei MS: Genomic imprinting in mammals. Cold Spring Harb Perspect Biol 2014, 6.

27. Bartolomei MS, Ferguson-Smith AC: Mammalian genomic imprinting. Cold Spring Harb Perspect Biol 20I I, 3.
28. Xie W, Barr CL, Kim A, Yue F, Lee AY, Eubanks J, Dempster EL, Ren $B$ : Base-resolution analyses of sequence and parent-oforigin dependent DNA methylation in the mouse genome. Cell 20I2, I48:8I6-3I.

\section{FlOOOPrime
RECOMMENDED}

29. Khatib $\mathrm{H}$ : Is it genomic imprinting or preferential expression? Bioessays 2007, 29:1022-8.

\section{FlOOOPrime}

\section{RECOMMENDED}

30. Gregg C, Zhang J, Weissbourd B, Luo S, Schroth GP, Haig D, Dulac C: High-resolution analysis of parent-of-origin allelic expression in the mouse brain. Science 2010, 329:643-8.

\section{FlOOOPrime
RECOMMENDED}

31. Babak T, Deveale B, Armour C, Raymond C, Cleary MA, van der Kooy, Derek, Johnson JM, Lim LP: Global survey of genomic imprinting by transcriptome sequencing. Curr Biol 2008, 18: $1735-4 \mid$.

\section{FlOOOPrime}

32. Wang X, Sun Q, McGrath SD, Mardis ER, Soloway PD, Clark AG: Transcriptome-wide identification of novel imprinted genes in neonatal mouse brain. PLoS ONE 2008, 3:e3839.

33. Wang $X$, Soloway PD, Clark AG: A survey for novel imprinted genes in the mouse placenta by mRNA-seq. Genetics 201I, 189: 109-22.

34. Menheniott TR, Woodfine K, Schulz R, Wood AJ, Monk D, Giraud AS, Baldwin HS, Moore GE, Oakey RJ: Genomic imprinting of Dopa decarboxylase in heart and reciprocal allelic expression with neighboring Grbl0. Mol Cell Biol 2008, 28:386-96.

35. Deveale B, van der Kooy, Derek, Babak T: Critical evaluation of imprinted gene expression by RNA-Seq: a new perspective. PLoS Genet 2012, 8:el002600.

\section{FIOOOPRime}

36. Finn EH, Smith CL, Rodriguez J, Sidow A, Baker JC: Maternal bias and escape from $X$ chromosome imprinting in the midgestation mouse placenta. Dev Biol 2014, 390:80-92.

\section{FlOOOPrime
RECOMMENDED}

37. Ferrón SR, Charalambous M, Radford E, McEwen K, Wildner $\mathrm{H}$, Hind E, Morante-Redolat JM, Laborda J, Guillemot F, Bauer SR, Fariñas I, Ferguson-Smith AC: Postnatal loss of Dlkl imprinting in stem cells and niche astrocytes regulates neurogenesis. Nature $20 I I, 475: 38 I-5$.

\section{FlOOOPrime} RECOMMENDED

38. Judson MC, Sosa-Pagan JO, Del Cid, Wilmer A, Han JE, Philpot BD: Allelic specificity of Ube $3 a$ expression in the mouse brain during postnatal development. J Comp Neurol 20 14, 522:1874-96.

\section{FlOOOPrime
RECOMMENDED}

39. Yamasaki K, Joh K, Ohta T, Masuzaki H, Ishimaru T, Mukai T, Niikawa N, Ogawa M, Wagstaff ], Kishino T: Neurons but not glial cells show reciprocal imprinting of sense and antisense transcripts of Ube3a. Hum Mol Genet 2003, I2:837-47.

\section{FlOOOPrime}

\section{RECOMMENDED}

40. Wilkinson LS, Davies W, Isles AR: Genomic imprinting effects on brain development and function. Nat Rev Neurosci 2007, 8:832-43

4I. Blagitko N, Mergenthaler S, Schulz U, Wollmann HA, Craigen W, Eggermann T, Ropers HH, Kalscheuer VM: Human GRBIO is 
imprinted and expressed from the paternal and maternal allele in a highly tissue- and isoform-specific fashion. Hum Mol Genet 2000, 9:1587-95.

\section{FlOOOPrime}

\section{RECOMMENDED}

42. Hu JF, Oruganti H, Vu TH, Hoffman AR: Tissue-specific imprinting of the mouse insulin-like growth factor II receptor gene correlates with differential allele-specific DNA methylation. Mol Endocrinol 1998, I2:220-32.

\section{FlOOOPRime
RECOMMENDED}

43. Hetts SW, Rosen KM, Dikkes P, Villa-Komaroff L, Mozell RL: Expression and imprinting of the insulin-like growth factor II gene in neonatal mouse cerebellum. J Neurosci Res 1997, 50:958-66.

\section{FlOOOPrime}

44. Kaneko R, Kato H, Kawamura $Y$, Esumi S, Hirayama T, Hirabayashi T, Yagi T: Allelic gene regulation of Pcdh-alpha and Pcdh-gamma clusters involving both monoallelic and biallelic expression in single Purkinje cells. J Biol Chem 2006, 28 I:3055 I-60.

45. Wood AJ, Schulz R, Woodfine K, Koltowska K, Beechey CV, Peters J, Bourc'his D, Oakey RJ: Regulation of alternative polyadenylation by genomic imprinting. Genes Dev 2008, 22: | |4|-6.

\section{FlOOOPrime RECOMMENDED}

46. Choi JD, Underkoffler LA, Wood AJ, Collins JN, Williams PT, Golden JA, Schuster EF, Loomes KM, Oakey RJ: A novel variant of Inpp 5f is imprinted in brain, and its expression is correlated with differential methylation of an internal CpG island. Mol Cell Biol 2005, 25:55I4-22.

47. Wood AJ, Roberts RG, Monk D, Moore GE, Schulz R, Oakey RJ: A screen for retrotransposed imprinted genes reveals an association between $\mathbf{X}$ chromosome homology and maternal germ-line methylation. PLoS Genet 2007, 3:e20.

\section{FlOOOPrime}

48. Miyanari Y, Torres-Padilla M: Control of ground-state pluripotency by allelic regulation of Nanog. Nature 2012, 483:470-3.

FIOOOPRime
RECOMMENDED

49. Lawson HA, Cheverud JM, Wolf JB: Genomic imprinting and parent-of-origin effects on complex traits. Nat Rev Genet 2013, 14:609-17.

50. Mott R, Yuan W, Kaisaki P, Gan X, Cleak J, Edwards A, Baud A, Flint J: The architecture of parent-of-origin effects in mice. Cell 2014, I 56:332-42.

5I. Hansen $\mathrm{CH}$, van Oudenaarden A: Allele-specific detection of single mRNA molecules in situ. Nat Methods 2013, 10:869-71.

\section{FIOOOPrime
RECOMMENDED}

52. Levesque MJ, Ginart P, Wei $Y$, Raj A: Visualizing SNVs to quantify allele-specific expression in single cells. Nat Methods 2013, 10:865-7.

\section{FlOOOPrime}

53. Miller MR, Robinson KJ, Cleary MD, Doe CQ: TU-tagging: cell type-specific RNA isolation from intact complex tissues. Nat Methods 2009, 6:439-4I.

\section{FlOOOPrime}

54. Heiman M, Schaefer A, Gong S, Peterson JD, Day M, Ramsey KE, Suárez-Fariñas M, Schwarz C, Stephan DA, Surmeier DJ, Greengard P, Heintz N: A translational profiling approach for the molecular characterization of CNS cell types. Cell 2008, 135:738-48.

\section{FlOOOPrime}

\section{RECOMMENDED}

55. Doyle JP, Dougherty JD, Heiman M, Schmidt EF, Stevens TR, Ma G, Bupp S, Shrestha P, Shah RD, Doughty ML, Gong S, Greengard P, Heintz N: Application of a translational profiling approach for the comparative analysis of CNS cell types. Cell 2008, 135:749-62.

FlOOOPrime
RECOMMENDED

